\title{
Photo-DSC Investigation of Acetonaphthone Type Photoinitiators at Different Light Intensities
}

\author{
Sevnur Keskin Dogruyol* \\ Department of Chemistry, Yildiz Technical University, Davutpasa Campus, 34220 \\ Istanbul Turkey \\ *dogruyol@yildiz.edu.tr
}

\begin{abstract}
The gelation of a $75 \%$ epoxy diacrylate (EA) and $25 \%$ tripropyleneglycol diacrylate (TPGDA) mixture (P-3038) was performed using 2-( $N$-methyl- $N$-phenyl)acetonaphthone (MPA) and morpholino acetonaphthone (MPM) as Type I photoinitiators at different light intensities by using a photo-differential scanning calorimetric (Photo-DSC) technique. Identical temperature values and initiator concentrations were used for the photopolymerization reactions. The results show that maximum rate of polymerization $\left(R \mathrm{p}_{\max }\right)$ and final conversion values present good correlation at different light intensities.

Keywords: Photopolymerization, Photoinitiator, Acetonaphthone, Photo-DSC, Light intensity
\end{abstract}

\section{Introduction}

Polymerization reactions of photoinitiated free radicals are extensively applied in many commercial areas. The processes of photoinitiated polymerization of acrylates and methacrylates provide a rapid production of polymeric materials. These materials are widely used as coatings, imaging materials, photoresists and polymeric materials in many applications for their welldefined properties [1-7].

Photoinitiators are capable of generating reactive species, e.g. free radicals or ions that are used in UV-curing systems and initiating polymerization of multifunctional monomers and oligomers. Their properties of in addition, the curing performance of a photoinitiator can be improved by a proper selection of polymerization rate $(R \mathrm{p})$, double bond conversion, and final adhesive properties [8,9]. Two types of photoinitiators are used in free radical polymerization: an $\alpha$-cleavable initiator (Type I) and a hydrogen abstraction type photoinitiator (Type II). Type II initiators are responsible for yielding the initiating radicals by reacting with the $\mathrm{H}$-donor compounds like tertiary amines, thiols, alcohols and ethers at the triplet state. Studies on this field have mostly focused on Type I photoinitiators, which produce two radical species by undergoing an $\alpha$-cleavage process upon irradiation $[1,10,11]$. Type I photoinitiators are highly reactive and they have very short triplet state lifetimes [12]. Therefore, the introduction of substituted $\alpha$-aminoketone photoinitiators constituted a great development in this field. Since their molar absorptivities in the range of 300-400 $\mathrm{nm}$ are higher than the usual acetophenone type photoinitiators, these compounds can be used in the curing of pigmented systems [3,12]. Upon irradiation these ketones undergo type I cleavage to produce radical pairs with high rate constant and good efficiency. The addition of radicals to alkenes is one of the most important reactions in free radical polymerization. These photoinitiators yield $\alpha$ aminoalkyl radicals as well as substituted benzoyl radicals. According to time-resolved IR spectroscopic studies, $\alpha$-aminoalkyl radicals are more efficient compare to benzoyl radicals in initiation of free radical polymerization [13].

Photo-DSC (Photo-Differential Scanning Calorimetry) technique is widely used to investigate different parameters of curing reactions such as the extent of crosslinking and conversion, the order of reactions and the rate of polymerization. With this technique, the change in the heat flow of the investigated system is observed and interpreted [14]. Photoinitiated polymerization of multifunctional

Received June 8,2017

Accepted August 9, 2017 
monomers yields crosslinked polymers which induce particular behaviors with regard to kinetic reactions. Light intensity, thickness of sample, absorption properties and concentration of initiator constitute the main determinant factors of the rate of initiation, as well as the number of initiating species that are produced for each photon of light. The effect of light intensity and exposure time on photopolymerization has been also explored by different scholars. Accordingly, UV light intensity was identified among the most important factors which affect the reaction kinetics of resin systems and properties of formed gels $[15,16]$.

In this study, 2-( $N$-methyl- $N$-phenyl $)$ acetonaphthone (MPA) and morpholino acetonaphthone (MPM) (Fig. 1) were synthesized and used as Type I photoinitiators for the polymerization of $75 \%$ epoxy diacrylate (EA) and $25 \%$ tripropyleneglycol diacrylate (TPGDA) mixture (P-3038). Photo-DSC technique is used to investigate photopolymerization kinetics at different light intensities in the presence of MPA and MPM under nitrogen atmosphere. A strong correlation was found between the applied UV light intensity and maximum conversion rate $\left(C_{\mathrm{Rp} \max }\right)$, maximum rate of polymerization $\left(R_{\mathrm{p} \max }\right)$, and final conversion values.

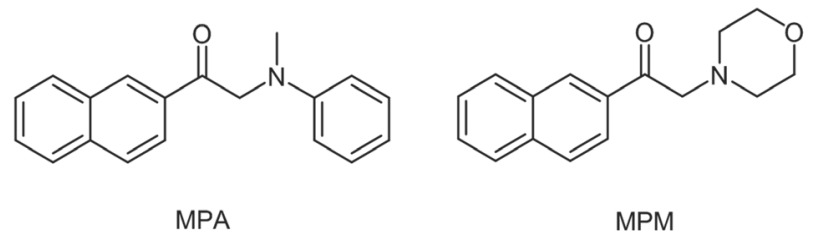

Fig. 1. Structures of MPA and MPM.

\section{Experimental}

2.1. Materials

The synthesis of 2-(N-methyl- $N$-phenyl) acetonaphthone (MPA) and morpholino acetonaphthone (MPM) were made based on the previously described procedure $[17,18]$. A mixture (P-3038) of 75\% epoxy diacrylate (EA) and 25\% tripropyleneglycol diacrylate (TPGDA) (P-3038) was provided from Cognis France. Irgacure 369, Irgacure 651 and Irgacure 907 were provided from Ciba Specialty Chemicals and used as received. Acetonitrile (99\% Merck) was used as received.

\subsection{Analysis}

A Varian UV-visible Cary 50 spectrophotometer was used for the analysis of UV-visible spectra. Photo-DSC experiments were performed using a
TA DSC 100 instrument and a TA Q PCA photo unit that were equipped with a high pressure mercury lamp.

\subsection{Photo-differential scanning calorimetry}

The heat released during the polymerization corresponds to the amount of reacted acrylates in the system. Formula 1 helps to specify the conversion of the acrylate groups $(C)$ or the extent of the reaction by integrating the area under exothermic peak:

$$
C=\Delta H t / \Delta H_{0}^{\text {theory }}
$$

In this formula, $\Delta H t$ represents the reaction heat evolved at time $t$ and $\Delta H_{0}^{\text {theory }}$ represents the theoretical heat for complete conversion. An acrylate double bond polymerization was carried out with a heat of $\Delta H_{0}{ }^{\text {theory }}=86 \mathrm{~kJ} / \mathrm{mol}$ [19]. A direct relationship was found between the rate of polymerization $(R \mathrm{p})$ and the heat flow $(\mathrm{d} H / \mathrm{d} t)$, which is represented by the general formula 2 :

$$
R \mathrm{p}=(\mathrm{d} C / \mathrm{d} t)(\mathrm{d} H / \mathrm{d} t) / \Delta H_{0}^{\text {theory }}
$$

Samples were accurately weighed into the aluminum pans and irradiated with the light source of the photo unit at different light intensities. The properties were measured in isothermal mode at $25^{\circ} \mathrm{C}$ under an inert atmosphere of nitrogen $(50 \mathrm{~mL}$ $\left.\min ^{-1}\right)$. The polymerization rates and conversion percentages were calculated.

\section{Results and discussion}

The structural analysis for the synthesis of cleavable photoinitiator of 2-( $N$-methyl- $N$-phenyl $)$ acetonaphthone (MPM) and morpholino acetonaphthone (MPM) were performed by spectral and elemental analysis. Both MPA and MPM exhibit good absorption properties with high molar absorptivities compared to commercial Type I photoinitiators, as shown in Fig. 2.

Photo-DSC is capable of indicating the performance of a photoinitiator accurately and fastly. Photopolymerization of P-3038 containing photoinitiators MPA and MPM under nitrogen atmosphere was observed through photo-DSC technique and the effect of light intensity on the rate of polymerization and conversion percentage was tested using four different light intensities. Figures 3-9 show time dependencies of the polymerization heat flow, polymerization rate and their conversion percentages for P-3038 formulations obtained from 
photo-DSC.

It was clearly seen that photopolymerization behaviour of MPM is different from that of MPA. In the sample containing MPM as photoinitiator UV light intensity increased from 10 to $60 \mathrm{~mW} \mathrm{~cm}^{-2}$, the extent of heat flow, rate of polymerization and conversion percentage values also increased in good order (Figs. 3-5). Most probably, increase of light intensity provides greater efficiency for initiation, leading to more formed primary radicals on the surface of the solution.

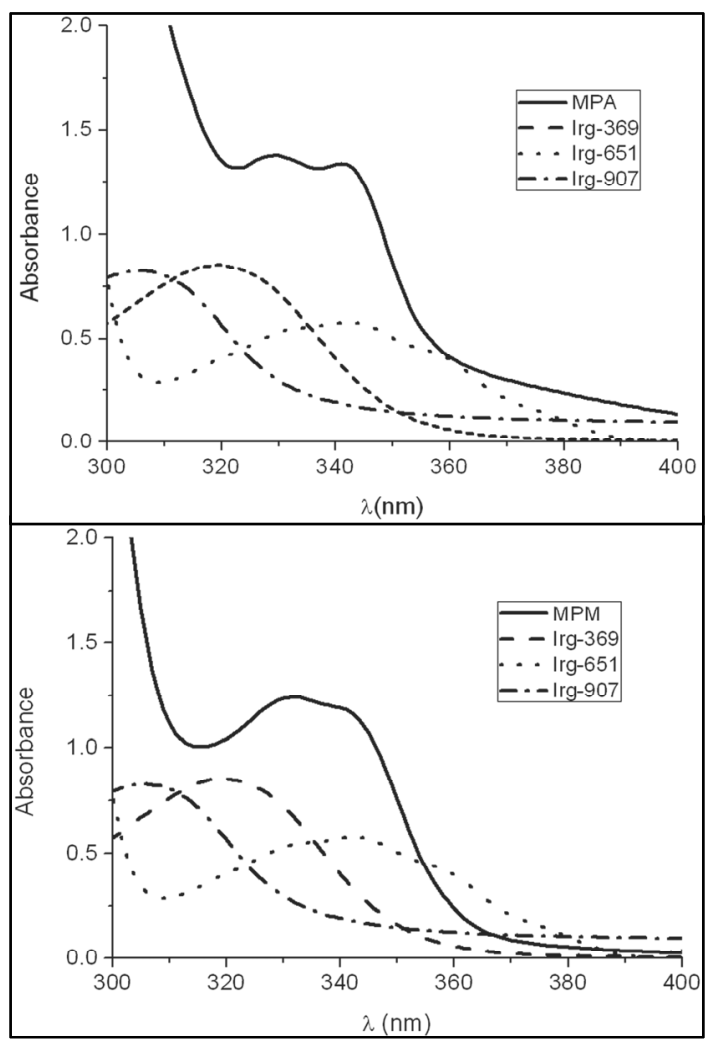

Fig. 2. Absorption spectra of $7.25 \times 10^{-4} \mathrm{M}$ MPA and MPM $\left(\lambda_{\max }: 329\right.$ and $332 \mathrm{~nm}, \varepsilon: 1840$ and 1733 $\mathrm{L} \cdot \mathrm{mol}^{-1} \mathrm{~cm}^{-1}$ ), Irgacure 369, Irgacure 651 and Irgacure 907 in acetonitrile.

Final conversion values increased properly with the increasing light intensity and reached $41.5 \%$ when the light intensity was $60 \mathrm{~mW} \cdot \mathrm{cm}^{-2}$ (Fig. 5, Table 1).

The conversion percentage of P-3038 to polymer reached $11.9 \%$ (Table 1) during $19.1 \mathrm{sec}$. of irradiation time with $60 \mathrm{~mW} \mathrm{~cm}^{-2}$ light intensity. The conversion was completed after nearly $300 \mathrm{sec}$ of irradiation with $41.5 \%$ of conversion percentage (Fig. 5. and Fig. 9).

Further experiments were carried out for the P3038 formulation with the same concentration of MPA at four different light intensities. For MPA,

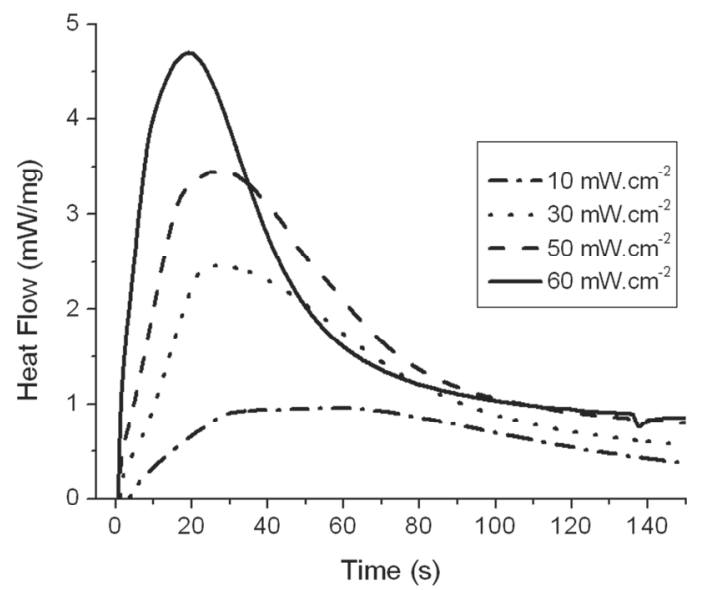

Fig. 3. Photopolymerization heat flow curves of EA/TPGDA with various UV light intensities in the presence of MPM as photoinitiator.

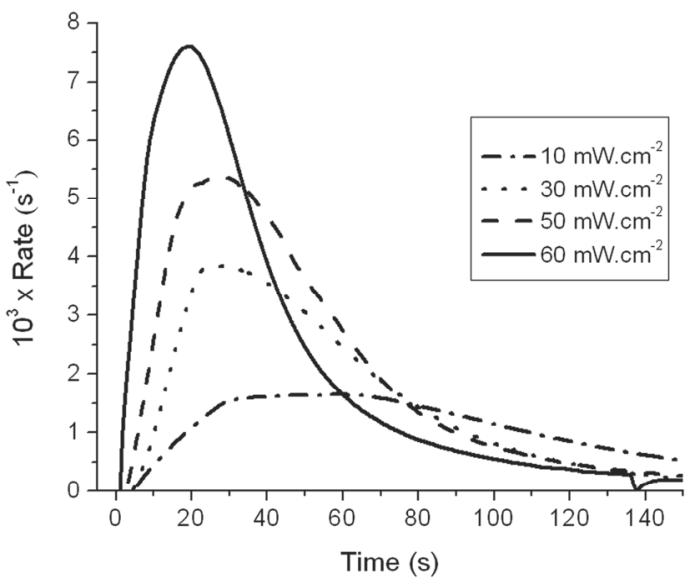

Fig. 4. Photopolymerization rate curves of EA/TPGDA with various UV light intensities in the presence of MPM as photoinitiator.

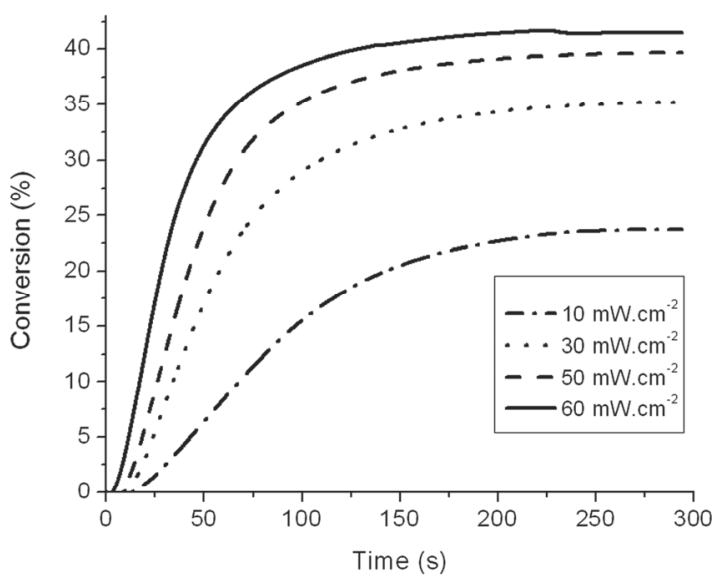

Fig. 5. Conversion spectra of photopolymerization of EA/TPGDA with various UV light intensities in the presence of MPM as photoinitiator. 
heat flow and polymerization rate was extended to $60 \mathrm{~mW} \cdot \mathrm{cm}^{-2}$ as the UV light intensity increased from 10 to $60 \mathrm{~mW} \mathrm{~cm}^{-2}$ (Figs. 6 and 7). However, an irregularity was observed in conversion percentages with the increasing light intensity (Fig. 8). Accordingly, monomer was converted to polymer with $39.8 \%$ conversion percentage at $40 \mathrm{~mW} \mathrm{~cm}^{-2}$. When light intensity reached $60 \mathrm{~mW} \cdot \mathrm{cm}^{-2}$, conversion percentage value of the formulation dropped to $38.8 \%$ (Figs. 8 and 9). It is of high possibility that high light intensity leads to a high cross-section for initiation, which results in production of more formed primary radicals on the surface of the solution. These excess radicals formed on the surface of the coating restricted the penetration of UV light into the samples by creating a filter effect, which resulted in a decrease of the final conversion values at high light intensities [1, $15,16]$.

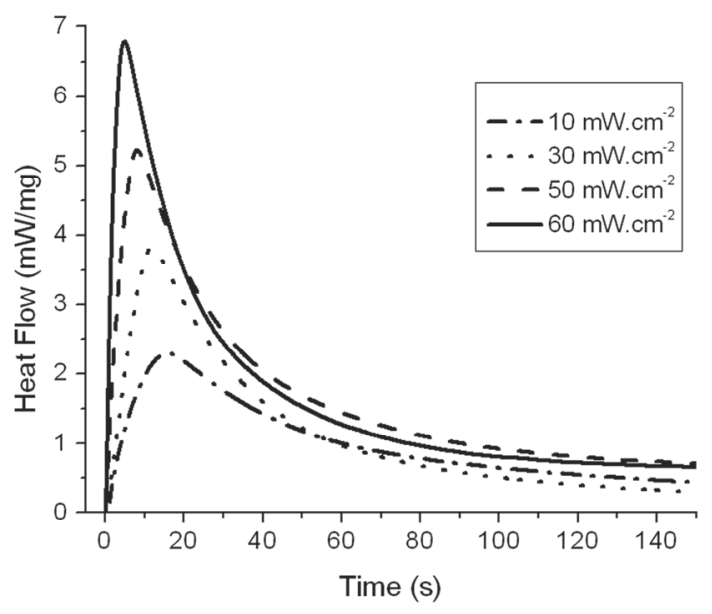

Fig. 6. Photopolymerization heat flow curves of EA/TPGDA with various UV light intensities in the presence of MPA as photoinitiator.

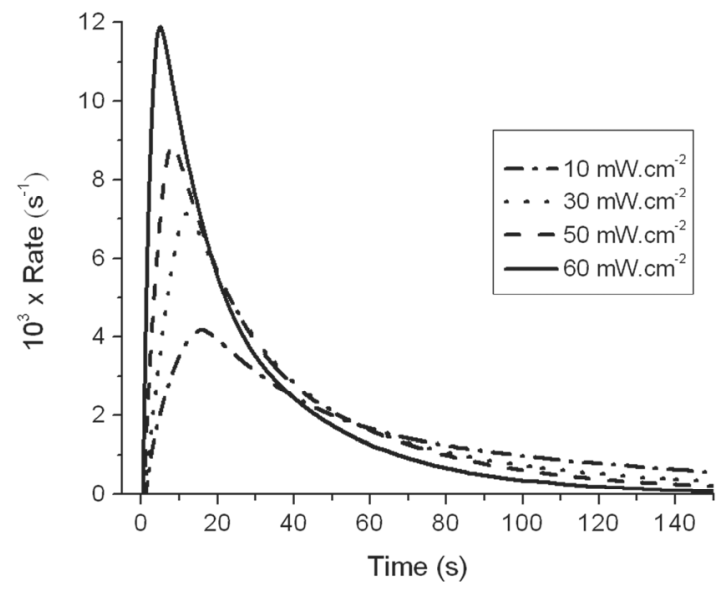

Fig. 7. Photopolymerization rate curves of EA/TPGDA with various UV light intensities in the presence of MPA as photoinitiator.

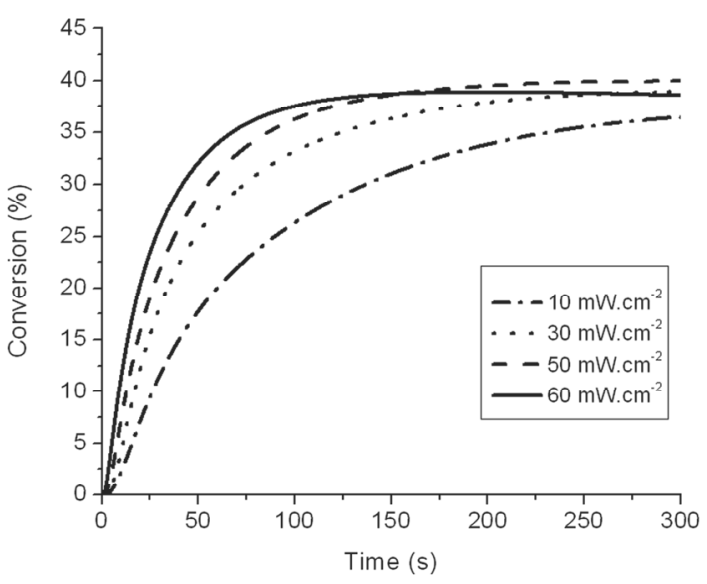

Fig. 8. Conversion spectra of photopolymerization of EA/TPGDA with various UV light intensities in the presence of MPA as photoinitiator.

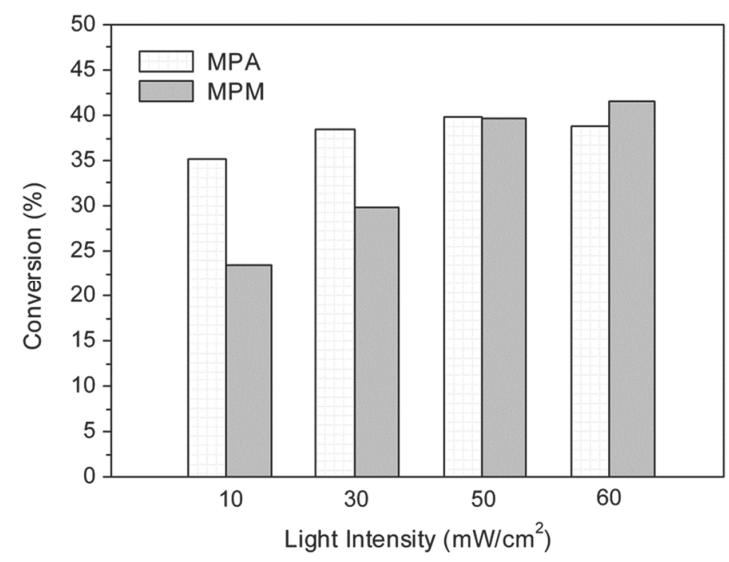

Fig. 9. Final conversion values of photopolymerization of EA/TPGDA with various UV light intensities in the presence of MPA and MPM as photoinitiators.

As can be seen in Figs. 3-8 and Table 1, because MPA polymerizes faster than MPM, the filter effect can be observed more clearly for MPA.

Table 1 shows $t_{\max }$ (time to reach polymerization rate), $R_{\text {pmax }}$ (maximum polymerization rate) and $C_{\text {Rpmax }}$ (maximum conversion rate) values versus four light intensities during EA/TPGDA photopolymerization.

According to the results of Table 1, for both initiators, when light intensity was increased, $t_{\max }$ values dropped and polymerization rates expectedly increased. When the light intensity reached the maximum value, maximum polymerization rate increased up to $11.9 \mathrm{~s}^{-1}$ and MPM reached $7.6 \mathrm{~s}^{-1}$ rate value during 19.1 seconds of irradiation. On the other hand, there was an adverse effect between final conversion values of MPA and MPM. Since polymerization of MPA occurred faster than MPM, final conversion values of MPM were less for the 
Table 1 . The values of various parameters measured using photo-DSC for different light intensities during diacrylate, EA/TPGDA photopolymerization.

\begin{tabular}{ccrrr}
\hline PI & $\begin{array}{c}\text { Light Int. } \\
\left(\mathrm{mW} . \mathrm{cm}^{-2}\right)\end{array}$ & \multicolumn{1}{c}{$\begin{array}{c}t_{\max } \\
(\mathrm{s})\end{array}$} & $\begin{array}{r}10^{3} \cdot R_{\mathrm{pmax}} \\
\left(\mathrm{s}^{-1}\right)\end{array}$ & \multicolumn{1}{c}{$\begin{array}{c}C_{\mathrm{Rpmax}} \\
(\%)\end{array}$} \\
\hline MPA & 10 & 15.7 & 4.2 & 5.0 \\
MPM & 10 & 58.5 & 1.6 & 8.2 \\
MPA & 30 & 11.9 & 7.1 & 5.8 \\
MPM & 30 & 26.7 & 3.8 & 6.4 \\
MPA & 50 & 8.1 & 8.9 & 5.2 \\
MPM & 50 & 28.3 & 5.4 & 11.4 \\
MPA & 60 & 4.9 & 11.9 & 4.5 \\
MPM & 60 & 19.1 & 7.6 & 11.9 \\
\hline
\end{tabular}

light intensities 10,30 and $50 \mathrm{~mW} \cdot \mathrm{cm}^{-2}$. However, for $60 \mathrm{~mW} \cdot \mathrm{cm}^{-2}$, because of the filter effect on MPA, final conversion of MPM is higher than MPA (Fig. 9).

The type of initiator is an important parameter, which reveals the effect of oxygen inhibition on the photopolymerization reaction. In this study, we have used MPA and MPM as type I initiators. The oxygen inhibition was not harmful and also photoDSC experiments were carried out under nitrogen atmosphere.

\section{Conclusion}

In this study, photopolymerization of P-3038 monomer was investigated using two acetonaphthone type photoinitiators with different light intensities. The results show that rate of polymerization and final conversion values present good correlation at different light intensities. According to the results from our previous studies it was observed that photoinitiators MPA and MPM produce naphtoyl and $\alpha$-aminoalkyl radicals under UV irradiation [17,18] and when the polymerization of MMA is performed using MPA as the initiator, the conversion values are higher compared to MPM [17].

For photo-DSC results, MPA polymerizes faster than MPM and this shows us different $\alpha$-aminoalkyl radicals produce from the initiators have different initiation effects [20-22]. The increase of the rate of polymerization is dependent upon the structure of the $\alpha$-aminoalkyl radical. In the case of MPM, the morpholino group is a bulky substituent and addition rate constant of the $\alpha$-aminoalkyl radical to acrylate double bond is less compare to MPA. This is most probably due to steric effects. Besides aromatic $N$-methyl aniline substituent in MPA is probably resonance stabilized and $\alpha$-aminoalkyl radical is more stable and efficient on addition on acrylate double bond. However, previous studies have shown these radicals efficiency of fast curing of acrylates [22].

Polymerization mechanism and efficiencies of $\alpha$-aminoalkyl radicals can be investigated in detail by ESR (electron spin resonance) and laser flash photolysis methods as advanced techniques $[12,23,24]$.

\section{Acknowledgements}

This study was conducted with the support of Yildiz Technical University, Scientific Research Projects Coordination Department. Project Number: 2011-01-02-08. I would like express my special thanks to TUBITAK, DPT and Prof. Dr. Nergis Arsu for providing me with her photo-DSC equipment and giving valuable suggestions.

\section{References}

1. R. S. Davidson, Exploring the Science. Technology and Applications of U.V. and E.B. Curing, SITA Technology Ltd.: London, (1999).

2. J. P. Fouassier, Photoinitiation, Photopolymerization and Photocuring: Fundementals and Applications, Henser Publishers: Munich Vienna New York, (1995).

3. K. Dietliker, In Chemistry \& Technology of UV \& EB Formulation for Coatings, Inks \& Paints, 2nd ed.; SITA Technology Ltd.: London, UK, (1998).

4. H. S. Hageman, Photopolymerization and Photoimaging Science and Technology, Elsevier: London, (1989).

5. S. P. Pappas, UV Curing Science and Technology, Technology Marketing Corp., Norwalk, CT, (1978).

6. K. Dietliker, Chemistry \& Technology of $U V$ \& EB Formulation for Coatings, Inks \& Paints, Vol. III, SITA Technology Ltd, London, (1991).

7. X. Jiang and J. Yin, J. Appl. Polym. Sci., 94 (2004) 2395.

8. M. K Mishra and Y. Yagci, Handbook of Radical Vinyl Polymerization, Marcel Dekker, New York, (1998), Chap. 7. 
9. G. Ullrich, B. Ganster, U. Salz, N. Moszner, and R. Liska, J. Polym. Sci. Part A: Polym. Chem., 44 (2006) 1686.

10. J. P. Fouassier and J. Lalevee, Photoinitiators for Polymer Synthesis: Scope, Reactivity and Efficiency, Wiley-VCH, Weinheim, (2012).

11. S. Dadashi-Silab, C. Aydogan, and Y. Yagci, Polym. Chem., 6 (2015) 6595.

12. S. Jockusch and N. J. Turro, J. Am. Chem. Soc., 121 (1999) 3921.

13. C. S. Colley, D. C. Grills, N. A. Besley, S. Jockusch, P. Matousek, A. W. Parker, M. Towrie, N. J. Turro, P. M. W. Grill, and M. W. George, J. Am. Chem. Soc., 124 (2002) 14952.

14. Z. Dogruyol, F. Karasu, D. K Balta, N. Arsu, and Ö. Pekcan, Phase Trans., 81 (2008) 935.

15. Z. Dogruyol, N. Arsu, and Ö. Pekcan, J. Macromol. Sci. Part B: Phys., 48 (2009) 74.

16. Z. Dogruyol, N. Arsu, S. K. Dogruyol, and Ö. Pekcan, Prog. Org. Coat., 72 (2011) 763.
17. S. Keskin and N. Arsu, Polym. Bull., 57 (2006) 643.

18. S. Keskin and N. Arsu, Prog. Org. Coat., 57 (2006) 348.

19. E. Andrejewska and M. Andrzejewski, $J$. Polym. Sci. Part A: Polym. Chem., 36 (1998) 665.

20. N. Arsu and R. S. Davidson, J. Photochem. Photobiol. A: Chem., 84 (1994) 291.

21. N. Arsu and M. Aydın, Die Angew. Makromol. Chem., 266 (1999) 70.

22. N. Arsu, J. Photochem. Photobiol. A: Chem., 153 (2002) 129.

23. M. Sakamoto, X. Cai, M. Hara, M. Fujitsuka, and T. Majima, J. Am. Chem. Soc., 126 (2004) 9709.

24. N. J. Turro, Modern Molecular Photochemistry, University Science Books, N.Y. (1991). 